\title{
A IMPORTÂNCIA DA GESTÃO DE CUSTOS EM HOSPITAIS DE ENSINO - CONSIDERAÇÕES TEÓRICAS
}

\author{
THE REAL IMPORTANCE OF COST MANAGEMENT IN A TEACHING \\ HOSPITAL - THEORETICAL CONSIDERATIONS
}

Maria Eulália L. V. Dallora', Aldaisa C. Forster ${ }^{2}$

\begin{abstract}
${ }^{1}$ Diretora da Assessoria Técnica do Hospital das Clínicas da Faculdade de Medicina de Ribeirão Preto - USP. ${ }^{2}$ Docente do Departamento de Medicina Social da Faculdade de Medicina de Ribeirão Preto - USP

CoRRESPondência: Maria Eulália Lessa do Valle Dallora. Assessoria Técnica. Hospital das Clínicas da Faculdade de Medicina de Ribeirão Preto - USP. Campus Universitário. 14048-900 Ribeirão Preto. meulalia@hcrp.fmrp.usp.br
\end{abstract}

Dallora MELV, Forster AC. A importância da gestão de custos em hospitais de ensino - considerações teóricas. Medicina (Ribeirão Preto) 2008; 41 (2): 135-42.

RESUMO: O objetivo deste trabalho é discutir as características dos hospitais de ensino e o papel que a gestão de custos assume no contexto dessas instituições de saúde.

O aumento dos gastos e custos na saúde é acompanhado com preocupação pelos gestores. O conhecimento dos custos é passo fundamental para a gestão dos hospitais, que estão inseridos em um contexto de custos elevados, recursos escassos, pressão por qualidade e bons serviços.

Nos hospitais de ensino, organizações complexas, a gestão dos custos vem assumindo grande importância, pois além da assistência à saúde, têm a importante missão de formação de recursos humanos.

Descritores: Hospitais de Ensino. Custos Hospitalares. Administração Hospitalar. Administração Financeira de Hospitais.

\section{1- INTRODUÇÃO}

A economia da saúde assume, cada vez mais, papel relevante em todo mundo. A limitação dos recursos utilizados na prestação da atenção médica e a demanda crescente das necessidades de saúde justificam estudos e práticas nesta área.

Paralelamente, a elevação dos gastos e custos com a assistência médica é uma tendência explicada por fatores como aumento e envelhecimento populacional, complexidade tecnológica, modificação do perfil epidemiológico com o surgimento de novas doenças e aumento da prevalência de moléstias crônico-degenerativas e do trauma, extensão da cobertura, papel reduzido do mercado e aumento do grau de consciência de cidadania com a população cada vez mais exigente em relação a seus direitos. ${ }^{1,2,3}$
Também merece destaque o processo gerencial tradicionalmente adotado que carece de informações de custos e de uma mentalidade de otimização das atividades, privilegiando a defesa da quantidade de recursos, sem contudo considerar a busca pela eficiência. $^{4}$

Em meio a um cenário mais competitivo, as organizações de saúde passam a ter necessidade de instrumentos de gestão econômica, sem os quais não conseguirão sua viabilização, para não dizer manutenção e sobrevivência. ${ }^{5}$

A avaliação econômica da saúde assume um papel de destaque, exigindo dos gestores o enfrentamento de novos desafios na busca contínua da eficiência e eficácia das atividades. A qualidade associada à utilização racional dos recursos deve ser o novo desafio. 
No entanto, se não existe uma cultura de medir custos e resultados o discurso da eficiência é vazio. ${ }^{6}$

Nos hospitais universitários - HUs estas questões aparecem com grande complexidade. Além da prestação da assistência médica, agregam atividades de ensino e pesquisa e, ainda, devem ser um referencial em tecnologia de ponta. Esses hospitais possuem características que incrementam seus custos em relação aos professados pelos não universitários, tais como: atividades docente-assistenciais e serviços básicos e secundários de saúde, quando sua estrutura é constituída para oferecer atividades de alta tecnologia. ${ }^{7}$

$\mathrm{O}$ volume de recursos para manter uma estrutura complexa como a dos HU's exige um rigoroso controle por parte dos gerentes, visando o processo de gestão da unidade hospitalar no contexto de sistema de saúde, ademais de incluir em suas atividades a otimização dos serviços com menores custos.

Neste sentido, o presente trabalho tem por objetivos apresentar e discutir as características dos hospitais universitários e de ensino e o papel que a gestão de custos assume no contexto dessas instituições de saúde para a necessária melhoria do processo de gestão.

Justifica-se o enfoque nos hospitais universitários e de ensino, além da complexidade dos serviços prestados, pelo grande aporte de recursos públicos necessários ao seu funcionamento e pelo que representam na produção da assistência médica brasileira. Conforme matéria da Gazeta Mercantil, a iniciativa privada vem perdendo espaço no Sistema Único de Saúde, ao passo que os hospitais públicos, especialmente os universitários, estão avançando ${ }^{8}$. Sem dizer que o modelo de gestão adotado por esses hospitais deve ser de excelência e servir de exemplo para demais unidades e aos profissionais de saúde, especialmente pelo seu caráter de ensino.

A metodologia empregada para a condução desse artigo foi a revisão bibliográfica da produção científica nacional sobre o tema.

\section{2- HOSPITAIS DE ENSINO}

A literatura sobre o assunto apresenta tanto a terminologia hospitais universitários quanto hospitais de ensino.

Segundo Bittar ${ }^{9}$, por hospital de ensino entende-se hospital universitário de propriedade ou gestão de universidade; hospital-escola de propriedade ou gestão de escolas médicas; hospital auxiliar de ensino que desenvolve programas de treinamento em serviço na área da saúde, devidamente conveniado com uma instituição de ensino superior.

Hospital universitário é tradicionalmente definido como sendo um prolongamento de um estabelecimento de ensino em saúde (faculdade de medicina, por exemplo); provedor de treinamento universitário na área da saúde; reconhecido oficialmente como hospital de ensino e prestador de atendimento médico de maior complexidade (nível terciário). ${ }^{7}$

Segundo definição do Ministério de Educação $(\mathrm{MEC})^{10}$, hospitais universitários são unidades de saúde, únicas em algumas regiões do país, capazes de prestar serviços altamente especializados, com qualidade e tecnologia de ponta à população. Garantem também, suporte técnico necessário aos programas mantidos por diversos Centros de Referência Estaduais ou Regionais e à gestão de sistemas de saúde pública, de alta complexidade e custos operacionais.

No Brasil, os hospitais de ensino foram assim denominados pela Portaria SNES/MS n ${ }^{\circ} 15$, de 8 de janeiro de 1991, que estabeleceu para estas instituições o Fator de Incentivo ao Desenvolvimento do Ensino e da Pesquisa Universitária em Saúde (FIDEPS). Hospitais de Ensino eram aqueles reconhecidos pelo MEC, funcionando regularmente há mais de 5 anos e pertencendo ao Sistema Integrado de Procedimentos de Alta Complexidade (SIPAC), do Ministério da Saúde (MS), como centro de referência nacional Ministério da Saúde. ${ }^{11}$

Em 2004, foi instituído um novo processo de certificação de hospitais de ensino que passaram a fazer jus a uma nova modalidade de contratação com o Sistema Único de Saúde (SUS). A Portaria Interministerial MEC-MS $n^{\circ} 1000$ de 15 de abril de $2004^{12}$ define então os novos requisitos para a certificação e estabelece como hospital de ensino as instituições hospitalares que servem de campo para a prática de atividades curriculares na área da saúde, sejam hospitais gerais ou especializados. Em suas considerações, a Portaria ressalta que:

[...] as Instituições de Ensino Superior, na área da saúde, têm, nas diretrizes curriculares nacionais, a determinação de contemplar, na formação dos profissionais, o sistema de saúde vigente no País, com atenção integral a saúde num sistema regionalizado e hierarquizado de referência e contra-referência, tendo como base o trabalho em equipe, com ênfase o Sistema Único de Saúde. ${ }^{12}$ 
A nova modalidade de contratualização dos hospitais de ensino envolve a definição de metas pactuadas com os gestores locais do SUS e uma nova regra de remuneração global com base no cumprimento das metas estabelecidas. Neste aspecto, é evidente a necessidade de melhoria da gestão dessas instituições que devem apresentar eficiência no uso dos recursos, devendo para tal, no mínimo, saber o quanto custam os procedimentos realizados.

Segundo a Associação Brasileira de Hospitais Universitários e de Ensino ${ }^{13}$, em 2003 o MEC e o MS tinham cadastrados 148 hospitais de ensino, representando em números aproximados, $2 \%$ do total de hospitais, $9 \%$ dos leitos, $12 \%$ das internações e $23 \%$ dos recursos aplicados em pacientes internados pelo SUS.

Estas informações, por si, são esclarecedoras da importância dos hospitais de ensino tanto no aspecto econômico quanto para as políticas públicas de saúde, já que a organização da atenção por eles prestada interfere de forma determinante sobre os demais prestadores, vez que são unidades de referência para todo o sistema de saúde.

Os hospitais de ensino são instituições caras, por contemplarem atendimentos de alta complexidade, além de atividades que mesclam assistência médica com procedimentos didáticos. Na Austrália o custo dos HUs é $12 \%$ mais elevado que de hospitais não universitários de alta tecnologia, enquanto que na Coréia do Sul esta parcela sobe para $28 \%$ quando comparados com hospitais de mesma complexidade. ${ }^{7}$

Como os HUs estão pouco integrados aos demais níveis de atenção e têm, em geral, total autonomia gerencial em relação aos sistemas de saúde, acabam atendendo a todos os níveis de atenção. Na verdade, são mais caros também por isto e não somente por incorporarem atividades docente-assistenciais. ${ }^{7}$

Hospitais são unidades complexas do ponto de vista gerencial. Conforme Mintzberg ${ }^{14}$, hospitais são estruturados segundo uma Burocracia Profissional que admite para o núcleo operacional, especialistas treinados. Alguns profissionais descrevem tais organizações como pirâmides invertidas, com os operadores profissionais no topo e os administradores abaixo para servi-los.

Segundo Cecílio $^{15}$, o hospital é a mais complexa das organizações de saúde. Este autor comenta duas antinomias fortemente presentes nos hospitais: a primeira é o grau de especialização e autonomia dos profissionais, em especial os médicos, versus a necessidade de coordenação das ações como um todo e a outra, é a tensão entre o poder técnico dos médicos e profissionais de saúde, centrado na base, com o poder administrativo. $\mathrm{O}$ autor destaca que o enfrentamento dessas questões deve passar pela mudança da gestão verticalizada, que é a mais tradicionalmente utilizada.

Conforme Campos ${ }^{16}$, o poder nos hospitais universitários está muito esfacelado, dentre outras razões, pela ordenação universitária por departamentos potencializada pela lógica médica de se organizar em dezenas de especialidades. Estas questões acabam por constituir instituições difíceis de governar.

Uma questão de primordial importância, relativa às unidades prestadoras de saúde inseridas no SUS, e aos hospitais de ensino em particular, é que não se tem total controle sobre as receitas, ou seja, o preço do serviço prestado que determina a receita SUS não é negociado e sim estabelecido pelo MS, até porque, a grande maioria dos hospitais não dispõe de estimativas de valores de custos para esta negociação. Os recursos SUS representam parcela representativa do financiamento hospitalar e sua tabela de remuneração por procedimento, não permite a cobertura integral dos custos de um hospital. Conforme Abrahue ${ }^{17}$ estudos realizados por alguns hospitais universitários indicam que a tabela SUS sub-remunera muitos procedimentos.

Hospitais universitários são caros e seu equilíbrio financeiro é um grande desafio que depende da receita e dos custos. Diante desse quadro, onde a receita é pouco negociada, uma possível solução passa pela gestão dos custos internos.

A gestão de custos nos hospitais universitários assume singular complexidade pela heterogeneidade e pouca padronização dos serviços prestados. Em função dessa complexidade muitos estudos fazem-se necessários e tem sido crescente, ainda que pouca, a atenção de pesquisadores nesta área. ${ }^{18}$

A natureza do trabalho do hospital é o que o diferencia do contexto das demais áreas de prestação de serviços. O trabalho de aliviar o sofrimento e preservar a saúde e a vida é difícil ser contextualizado como área de negócio. A harmonia entre o pensamento gerencial e a concepção médica da instituição hospitalar é o cenário atual no qual se expõe o hospital. ${ }^{1}$

Diminuir custos em hospitais é uma tarefa delicada pela própria natureza dos serviços prestados. Apesar dessas dificuldades, o gerenciamento de custos hospitalares, com vistas à otimização dos recursos, é necessário e um importante fator para o tão almejado equilíbrio financeiro e o aumento da qualidade da atenção prestada. 


\section{3- ECONOMIA DA SAÚDE}

Tradicionalmente, as profissões de saúde concentram-se na ética individualista, segundo a qual a saúde não tem preço e uma vida salva justifica qualquer esforço. Por outro lado, a economia fixa-se na ética do bem comum ou ética do social. Basicamente essas diferenças dizem respeito à utilização de recursos. Com isso, muito se debate acerca do conflito entre a ética da saúde e a ética econômica. ${ }^{19}$

Alguns conceitos de economia aliados aos da assistência à saúde têm ajudado nesta discussão que, cada vez mais ganha força, considerando a desproporção entre recursos disponíveis e as necessidades da sociedade, em especial as necessidades de saúde. Assim, as decisões em saúde podem e devem ser orientadas por princípios econômicos que constituem o fundamento da economia da saúde.

Segundo Del Nero ${ }^{19}$, a economia da saúde:

[...] tem por objetivo a otimização das ações de saúde, ou seja, o estudo das condições ótimas de distribuição de recursos disponíveis para assegurar à população a melhor assistência à saúde e o melhor estado de saúde possivel, tendo em conta meios e recursos limitados.

O conceito de economia da saúde leva em consideração a realidade atual de escassez e da insuficiência dos recursos para as necessidades de saúde.

Aos aspectos que tradicionalmente são considerados na medicina, por ocasião da avaliação das intervenções realizadas, quais sejam, a segurança do procedimento, a eficácia e a efetividade, um quarto aspecto é acrescentado, o da eficiência. ${ }^{20}$

Enquanto a eficácia trata dos resultados das intervenções em condições ideais e a efetividade dos resultados no dia a dia da população, a eficiência acrescenta um ingrediente econômico, ou seja, estamos obtendo o melhor benefício pelos recursos que estamos usando?

Acrescentar o critério de eficiência não significa contrapor-se a outros objetivos sanitários e de solidariedade. Melhorar a eficiência permite alcançar maiores níveis de saúde e de solidariedade a partir de recursos determinados. ${ }^{21}$

Segundo Couttolenc ${ }^{22}$, a avaliação econômica das políticas e ações de saúde não implica a predominância desta dimensão sobre as demais. Acrescenta que esta dimensão não pode ser ignorada e sim é parte integrante do processo decisório. O dinheiro disponível para a saúde é limitado e, portanto, deve ser uti- lizado eficientemente e de maneira a maximizar o resultado obtido.

Um dos princípios básicos da economia da saúde é adotar medidas racionalizadoras, que propiciem redução dos custos sem que ocorram impactos negativos nos níveis de saúde, ou seja, aumentar a eficiência dos serviços, sem que haja prejuízos na eficácia e na efetividade. ${ }^{7}$

Sob a ótica da economia da saúde o conflito existente entre a ética da saúde e a ética da economia não existe, muito pelo contrário, ao perseguir a eficiência das ações, é possível fazer mais com os mesmos recursos, o que significa corroborar com os princípios de universalidade e integralidade do Sistema Único de Saúde (SUS) maximizando os benefícios para a sociedade.

Análise econômica em saúde trabalha com apuração de custos e dentre as principais técnicas adotadas têm-se: Custo-Benefício, Custo-Efetividade, Custo-Utilidade, Custo Mínimo e Custo de Oportunidade ou Custo Social.

O Custo-Benefício avalia se os benefícios resultantes de um programa compensam seus custos. Destina-se a avaliar a viabilidade econômica de projetos sociais, podendo aplicá-lo a um determinado programa, ou a vários alternativos, para compará-los em termos de sua rentabilidade social. Mostra a relação entre os custos e os benefícios diretos e indiretos gerados. ${ }^{23}$

Atribuir a um conjunto de benefícios um valor em dinheiro, além de limitado enquanto método, é bastante complicado, em termos práticos e embora este seja, teoricamente, o método de avaliação mais abrangente, na prática a análise de custo benefício tem utilização restrita. ${ }^{24}$

A análise de Custo Efetividade destina-se à escolha da melhor estratégia para atingir um determinado objetivo, ou seja, trata-se de estudo comparativo de alternativas para executar uma mesma ação ${ }^{23}$. Para tanto, faz-se necessário identificar e quantificar a meta, definição das diferentes estratégias para alcançá-la, o cálculo dos custos e da efetividade de cada meta e por fim a análise da relação custo efetividade.

$\mathrm{Na}$ análise de Custo Utilidade compara-se diferentes tratamentos destinados, fundamentalmente, a pacientes crônicos. A unidade de comparação é a relação custo/sobrevida, sendo esta última quantificada por uma unidade qualitativa, o Qualy ou Ano de Vida Ajustado por Qualidade (AVAQ), que leva em conta o tempo e a qualidade da sobrevida decorrente de diferentes tipos de intervenção ${ }^{23}$ 
A análise de custo mínimo compara alternativas de programas/projetos/ações de saúde, que apresentam o mesmo resultado e escolhe-se aquela com menor custo.

Em todos estes conceitos está implícito um conceito mais amplo que é o de custo de oportunidade, que incorpora a noção de que os recursos utilizados para uma intervenção/projeto não estarão mais disponíveis para outros. O custo de oportunidade ou custo social reflete a escassez de recursos.

Ainda é incipiente no Brasil a realização de avaliações econômicas nos serviços de saúde, mesmo as mais simples. A descrição de custos e a descrição de resultados praticamente inexistem. Para viabilizar a realização de qualquer tipo de estudo de avaliação econômica, o ponto de partida deve ser a implementação nas organizações de saúde de um sistema de custeio. ${ }^{25}$

\section{4- SISTEMAS DE CUSTEIO}

A Contabilidade de Custos foi desenvolvida com a preocupação de mensuração monetária dos estoques e resultados e não como um instrumento administrativo. A visão gerencial, como forma de auxilio na tomada de decisões não data de mais de algumas décadas, e por essa razão, ainda há muito a ser desenvolvido. Importante ressaltar que as regras e os princípios geralmente aceitos na contabilidade de custos foram criados e mantidos com a finalidade básica de avaliação de estoques e dos resultados e não para controle e decisões gerenciais. Por essa razão são necessárias certas adaptações quando se deseja desenvolver bem esse seu outro potencial. ${ }^{26}$

Contudo, há necessidade de cautela na implementação de sistemas de custos no que diz respeito às expectativas de seus resultados imediatos para o controle e decisão. Sistema não é somente um conjunto de normas, fluxos, papéis e rotinas, mas um conjunto de pessoas. Sistema de custos não é exceção e seu sucesso depende fundamentalmente das pessoas. ${ }^{26}$

Custeio significa apropriação dos custos e sistema de custeio diz respeito à forma de calcular os custos ou de apropriação dos custos aos produtos e serviços. ${ }^{26}$

Os custos são classificados quanto ao seu comportamento em relação ao volume de produção dos serviços em custos fixos e variáveis, e quanto à forma de apropriação aos produtos e serviços em custos diretos e indiretos.
Os custos fixos são aqueles que permanecem constantes, independente do volume de produção. Os custos variáveis mantêm relação direta com a produção, ou seja, crescem à medida que o volume de atividades aumenta.

Os custos diretos podem ser diretamente identificados ao produto ou serviço enquanto que os indiretos necessitam de algum critério de rateio para a sua devida apropriação.

Para atender diferentes necessidades foram desenvolvidos muitos sistemas de custeio sendo os principais: custeio por absorção, custeio direto e custeio baseado em atividades. ${ }^{4,5}$ É possível a adoção simultânea e harmoniosa de vários deles, visto que não são excludentes entre si, cada qual com um conjunto de informações específicas às funções de uma instituição de saúde.

As dificuldades para a implementação de um sistema de custos e a correspondente geração de informações relevantes ao processo de gestão compreendem fatores de naturezas diversas, ressaltando o fator humano como elemento fundamental para o sucesso.

Para a implantação de alguns sistemas de custeio, como o custeio por Absorção que é o mais tradicionalmente utilizado nos hospitais, torna-se necessário dividir a instituição em centros de custos.

Centros de custos são unidades administrativas homogêneas baseadas na estrutura organizacional da instituição, com área circunscrita de abrangência em termos de responsabilização e objetivos comuns. ${ }^{27}$

Os centros de custos podem ser classificados em produtivos ou especiais, auxiliares e administrativos.

Os centros de custos produtivos são representados pelas unidades de assistenciais e desenvolvem as atividades fins do hospital com a prestação de um serviço direto ao paciente. Os centros de custos auxiliares e administrativos correspondem aos serviços de apoio e às funções de natureza administrativas. Caracterizam-se pela prestação de serviços internos.

Os poucos textos que abordam custos na área da saúde no Brasil apresentam pontos comuns e que justificam a necessidade de generalizar uma metodologia de cálculo e gestão de custos. Os pontos relevantes são que a grande maioria dos profissionais de saúde e avaliadores pouco sabem sobre procedimentos de apuração e análise de custos; poucos órgãos públicos realizam estudos; as metodologias existentes carecem de adaptações e simplificações; utilização incipiente de análise de custos como instrumento de gestão, nos diferentes níveis da administração. ${ }^{28}$ 


\section{5- GERENCIAMENTO DE CUSTOS HOSPI- TALARES}

Gerenciamento de custos é o conjunto de ações que os gestores tomam para satisfazer os clientes enquanto, continuamente, reduzem e controlam os custos. ${ }^{29}$

Conforme Grabois ${ }^{30}$, p.82:

[...] Quando tratamos de gerência, nos referimos a um conjunto de técnicas e métodos de planejamento, direção e controle, visando um envolvimento e mobilização dos atores hospitalares na concretização dos objetivos da instituição. Tais funções são exercidas não apenas pelo diretor-geral, mas por todos os profissionais que exercem funções que visem direcionar as práticas dos diferentes atores.

Este autor coloca que a prática gerencial é tida como crítica e estratégica para o enfrentamento das demandas e desafios atuais e somente assim os hospitais poderão cumprir seu papel social, com uma assistência de qualidade, a custos adequados e de acordo com as necessidades da população. Não mais se admite que estes serviços operem com desperdícios de recursos e sem compromisso e sensibilidade para com os problemas dos usuários.

Para tanto, os hospitais devem ter um melhor conhecimento dos seus custos de funcionamento e dispor de sistemas com informações confiáveis e tempestivas para subsidiar a análise, tomada de decisão e adoção de medidas corretivas em todas as fases do gerenciamento.

Pesquisas realizadas apontam que os sistemas de custos existentes nos hospitais brasileiros são menos acurados que os existentes nas empresas e os poucos artigos sobre custos encontrados na literatura dedicam-se ao ambiente industrial ${ }^{18}$. Esta questão pode ser explicada pelo fato que o hospital é das empresas mais complexas de serem administradas. ${ }^{31}$

O melhor gestor de custos é quem conhece profundamente as atividades desenvolvidas na unidade e não quem reúne apenas os conceitos relacionados à contabilização dos custos. O conceito de custo deve ser compreendido, como sendo além de uma questão econômica, uma questão também comportamental. ${ }^{5}$

No entanto, os profissionais da área de saúde tendem a rejeitar qualquer aproximação com problemas administrativos, tendo como premissa a idéia de que controlar o custo da assistência pode acarretar diminuição de recursos e, conseqüentemente, perda de qualidade. ${ }^{32}$

Os custos são aspectos fundamentais da gerência. O esforço para melhorar a eficiência dos sistemas de saúde passa pelo denominado gerenciamento sistemático da assistência à saúde que tem em seus princípios a responsabilização dos profissionais de saúde pelo custo dos serviços prestados e também, que a prestação de serviços deve ser gerenciada no dia-adia. Este princípio encoraja uma utilização criteriosa e racional dos recursos tecnológicos e chama a atenção que não se podem desvincular as dimensões técnicas das financeiras. ${ }^{33}$

$\mathrm{O}$ gerenciamento adequado de materiais e medicamentos, desde o planejamento até o consumo pelos pacientes é aspecto importante dos custos hospitalares. Em geral, investimentos em estoques são elevados e constituem parte significativa dos custos hospitalares e por isso é de grande importância seu planejamento e manuseio. ${ }^{34}$

Neste aspecto, o uso de protocolos de atenção com padrões que fornecem guias de tratamento clínico e indicam os recursos essenciais necessários para desenvolvimento das ações, constitui-se em ferramenta útil de gestão que se traduz em parâmetros de referência, permitindo reduzir a diversidade de produtos e a reserva dos depósitos contribuindo, deste modo, para redução de custos.

No setor saúde e nos hospitais em particular, os objetivos de um sistema de contabilidade de custos, segundo a American Hospital Association (AHA) ${ }^{1}$ apud Falk (2001, p.16), deve contemplar os seguintes componentes:

[...] Uma base comum para comunicação, negociação, planejamento e gerência de todos os niveis de pessoal hospitalar e, também, entre hospitais e instituições regulamentadoras;

Uma metodologia de medição dos efeitos de alterações em intensidade e diversidade dos casos atendidos;

Um método de avaliação e medição de performance contra um plano prefixado;

Uma forma de prover a informação necessária para a gerência eficiente de recursos de todos os niveis. Padrões desenvolvidos nesse processo permitem aos gerentes prever gastos em recursos humanos e não pessoais baseados nos niveis de utilização dos serviços da instituição, em curtos periodos de tempo; 
Um método para facilitar a administração a identificar gastos que podem ser alterados de fixos para variáveis. Se o método for bem-sucedido, diminuições em volume serão menos críticas para o bem-estar financeiro do hospital;

Um método de identificar funções ineficientes e demonstrar qual a natureza do problema em termos de preço, volume ou prática organizacional. ${ }^{31}$

\section{6- CONSIDERAÇÕES FINAIS}

A gestão na área da saúde pública brasileira é um processo desafiador, vez que deve aliar fatores como o compromisso da prestação de serviços de qualidade a população e a escassez de recursos. Sendo assim, a preocupação tem sido a de adequar os escassos recursos disponíveis à assistência, sem prejuízo do usuário.

A implementação de um sistema de custeio é ferramenta útil para a gestão eficiente dos hospitais, que estão inseridos em um contexto de custos elevados, recursos escassos, pressão por qualidade e bons serviços. Em especial, os hospitais de ensino devem primar pela eficiência dos serviços prestados à socie- dade, pois além da assistência à saúde, têm a importante missão de formação de recursos humanos.

Importante ressaltar que a decisão dos bens e serviços a serem empregados no processo assistencial é de competência da equipe de saúde, em especial do médico. Assim o conhecimento gerencial de custos por esses profissionais é fator preponderante para o sucesso da implementação de qualquer sistema de custeio. Um novo modelo de gestão, com vistas a eficiência, a racionalização dos gastos e contenção dos custos deve considerar a integração das equipes de saúde e administrativa nas suas diferentes áreas do saber. ${ }^{35}$

A visão que deve prevalecer é a multidisciplinar, considerando principalmente, que na área da saúde os resultados não se medem simplesmente por ganhos econômicos.

Numa instituição de saúde, o resultado social é mais importante que o financeiro, mesmo assim, não se prescinde de um sistema de informações que permita ampla visão e gerenciamento dos custos das atividades desenvolvidas.

A gestão de custos ganha destaque, principalmente se considerarmos que recursos escassos bem gerenciados podem render mais. A conseqüência, imediata e mais importante, é maior qualidade e abrangência do sistema público de saúde.

Dallora MELV, Forster AC. The real importance of cost management in a teaching hospital - theoretical considerations. Medicina (Ribeirão Preto) 2008; 41 (2): 135-42.

ABSTRACT: The impact of rising Health Care Costs are observed with preoccupation by their managers. The knowledge of the costs is a basic step for the hospital management that are inserted in a context of raised costs, scarce resources, pressure for quality and good services. At teaching Hospitals a complex organization, the management of the costs comes assuming great importance, because besides the attendance to the health, they have the important mission of human resources formation.

Keywords: Hospitals, Teaching. Hospital Costs. Hospital Administration. Financial Management, Hospital.

\section{REFERÊNCIAS}

1 - Organização Pan-americana de Saúde. A transformação da gestão de hospitais na América Latina e Caribe. Brasília: OPAS/ OMS, 2004. 398p.

2 - Brasil. Ministério da Saúde. SCTIE/DES. Instituto de Pesquisa Econômica e Aplicada. DISOC. Projeto Economia da Saúde.
Atenção de Alta Complexidade no SUS: desigualdade de acesso e no financiamento. Volume I. Brasília; 2005. Disponível em: http://www.ipea.gov.br/economiadasaude/adm/arquivos/ destaque/alta_complexidade.pdf Acesso em: 01 fev. 2006.

3 - Médici AC. Economia e financiamento do setor saúde no Brasil: balanços e perspectivas do processo de descentralização. São Paulo: Faculdade de Saúde Pública/USP: AdSaúde; 1994. 
4 - Médici AC, Marques RM. Sistemas de custos como instrumento de eficiência e qualidade dos serviços de saúde. Cad FUNDAP 1996; p. 47-59.

5 - Matos AJ. Gestão de custos hospitalares. São Paulo: Editora STS; 2002. 280p.

6 - Médici AC. Economia política das reformas da saúde. Porto Alegre: IACHS; 1997.

7 - Médici AC. Hospitais universitários: passado, presente e futuro. Rev Assoc Med Bras 2001;47 (2):149-56.

8 - Gazeta Mercantil. O Mapa dos hospitais: empreendimentos privados perdem espaço. São Paulo, 27 jun 2006.

9 - Bittar OJNV. Instrumentos gerenciais para tornar eficiente o financiamento dos hospitais de ensino. Rev Adm Saúde 2002; 5 (17): 9-18.

10 - Brasil. Ministério da Educação. Hospitais universitários. Disponível em: http://www.portal.mec.gov.br/sesu/. Acesso em 30 jan. 2006.

11 - Brasil. Ministério da Saúde. Reforma do sistema da atenção hospitalar brasileira. Brasília: Ministério da Saúde, 2004. $164 p$.

12 - Brasil. Ministério da Saúde. Ministério da Educação. Certifica como hospital de ensino as instituições hospitalares que servirem de campo para a prática de atividades curriculares na área da Saúde. Portaria interministerial GM/MS n 1000 de 15 de abril de 2004. Diário Oficial da União. Brasília. Edição número 73 de 16/04/2004.

13 - Associação Brasileira de Hospitais Universitários e de Ensino. A reforma universitária e os hospitais universitários. 2004. Disponível em: http://www.abrahue.org.br/pdf/palestra_amancio.pdf Acesso em: 11 fev. 2006.

14 - Mintzberg H. Criando organizações eficazes: estruturas em cinco configurações. São Paulo: Atlas; 1995. 304p.

15 - Cecílio LCO. A modernização gerencial dos hospitais públicos: o difícil exercício da mudança. Rev Adm Pública 1997, 31(3): 36-47.

16 - Campos GWS. Educação médica, hospitais universitários e o Sistema Único de Saúde. Cad Saúde Pública (Rio de Janeiro) 1999 ; 15(1); 187-93.

17 - Associação Brasileira de Hospitais Universitários e de Ensino Hospitais Universitários e de Ensino no Brasil: Desafios e Soluções. 2005. Disponível em: http://www.abrahue.org.br/ Acesso em: 11 out. 2005.

18 - Miranda GJ, Carvalho CE, Martins VF, Faria AF. Custeio $A B C$ no ambiente hospitalar: Um estudo de caso nos hospitais universitários e de ensino brasileiros. Rev. Cont. Fin. USP São Paulo, no 44 p 33 - 43 maio/agosto 2007.

19 - Del Nero CR. O que é economia da saúde. In: Piola SF, Vianna SM. Economia da saúde: conceito e contribuição para a gestão da saúde. $3^{\mathrm{a}}$ ed. Brasília: IPEA; 1995. Cap I, p. 5-21.

20 - Ferraz MB. Como aproveitar os recursos disponíveis. Rev CREMESP 1996;17 (105): 8.

21 - Saes SG. Estudo bibliométrico das publicações em economia da saúde, no Brasil, 1989 - 1998. [Dissertação de Mestrado], São Paulo: Faculdade de Saúde Pública - USP; 2000. Disponível em: http://www.teses.usp.br/teses/disponiveis/ 6/6135/tde-01032002-132854/publico/suelisaes.pdf Acesso em: $23 / j a n / 2006$.
22 - Couttolenc BF. Por que avaliação econômica em saúde? Rev Assoc Med Bras 2001; 47(1). Disponível em: <http:// www.scielo.br/scielo.php?script=sci_arttext\&pid=S0104$42302001000100022 \&$ Ing=es\&nrm=iso $>$. Acesso em: 23 jan. 2005.

23 - Ugá MAD. Instrumentos de Avaliação Econômica dos Serviços de Saúde: Alcances e Limitações. In: . PIOLA, S. F.; VIANNA, S. M. Economia da Saúde: Conceito e Contribuição para a Gestão da Saúde. $3^{\text {a }}$ ed., Brasília: IPEA, 1995. Cap. IX. p. 209-26.

24 - Bevilácqua LDP. Farmacoeconomia. In: Gomes MJVM, Reis AMM. Ciências Farmacêuticas - Uma Abordagem em Farmácia Hospitalar. $1^{a}$ Ed., São Paulo: Ed. Atheneu, 2000. Cap. 11. p. 191-220.

25 - Castro JD. Instrumentos para avaliação econômica dos serviços de saúde. 2002. Doc em formato PDF. Disponível em: http://www.ipea.gov.br/economiadasaude/subtema.php? cod=16\# Acesso em: 07 out. 2005.

26 - Martins E. Contabilidade de custos. 9. ed., São Paulo: Atlas; 2003. 370p.

27 - Beulke R, Bertó J. Gestão de custos e resultado na saúde. $2^{\mathrm{a}}$ ed., São Paulo: Saraiva; 2000. 277p.

28 - Carpintéro JNC. Custos na área da saúde: considerações teóricas. In: Congresso Brasileiro de Custos, 6, 1999, São Paulo. Anais. São Paulo, 1999. cdroom

29 - Horngren CT, Foster G, Datar SM. Contabilidade de custos. $9^{a}$ ed., Rio de Janeiro: LTC - Livros Técnicos e Científicos; 2000. 701p.

30 - Grabois VA. implantação das técnicas de organização e gestão. In: Castelar R, Mordelet P, Grabois V. Gestão hospitalar: um desafio para o hospital brasileiro. Cooperação Brasil-França. Rio de Janeiro: Editions ENSP 1995. Cap. IV. p. 80-4.

31 - Falk JA. Gestão de custos para hospitais: conceitos, metodologias e aplicações. São Paulo: Atlas, 2001. 163p.

32 - Bittar E, Castilho V. O Custo médio direto do material utilizado em cirurgias de revascularização do miocárdio. Rev Assoc Med Bras 2003; 49 (3): 255-60. Documento em formato eletrônico. Disponível em: http://www.scielo.br/ scielo.php?script=sci_pdf\&pid=S0104-42302003000300027 \&lng=en\&nrm=is0\&tlng=pt. Acesso em: 8 abr. 2006.

33 - Couttolenc BF, Zucchi P. Gestäo de recursos financeiros. Säo Paulo: Instituto para o Desenvolvimento da Saúde / Universidade de Säo Paulo. Faculdade de Saúde Pública. Núcleo de Assistência Médico-Hospitalar / Banco Itaú; 1998. 120 p. (Saúde \& Cidadania, 10).

34 - Martins D. Custos e orçamentos hospitalares. São Paulo: Atlas; 2000. 165p.

35 - Dallora, MELV. Gerenciamento de custos de material de consumo em um hospital de ensino - Ribeirão Preto, 2007. 103 f. Dissertação (Mestrado em Saúde na Comunidade) - Faculdade de Medicina de Ribeirão Preto-USP.

Recebido para publicação em 14/09/2007.

Aprovado para publicação em 15/05/2008. 